\title{
Right Posterolateral Apical Peripheral Zone of Prostate
}

National Cancer Institute

\section{Source}

National Cancer Institute. Right Posterolateral Apical Peripheral Zone of Prostate. NCI

Thesaurus. Code C128582.

The region of the prostate that is located on the anatomical right side of the posterolateral portion of the apical division of the peripheral zone. 